\title{
Review
}

\section{Status and Progress in the Control of Infection in Chinese Hospitals}

\author{
Wenlong $\mathrm{He}$ \\ TianJin Medical Univercity School of Public Health, TianJin, China
}

\author{
Keywords \\ hospital-acquired infection; pathogenic \\ bacteria; management; cognition \\ Correspondence \\ Wenlong $\mathrm{He}$, \\ E-mail: hwltme_edu@163.com \\ DOI: 10.1515/ii-2017-0139
}

\begin{abstract}
This paper summarizes the characteristics and control of hospital-wide infections as identified by domestic surveys and research studies. This review also provides references to establish the basis for the control and management of hospital-acquired infection. Hospitalacquired infections are mainly initiated by Gram-negative bacteria and mainly localize in the lower respiratory tracts of patients. The intensive care unit has the highest infection rate among all hospital departments. Infants and the elderly are the most susceptible groups of patients to infection. Hands are an important route of pathogen transmission. The abuse of antibacterial drugs is an important factor of hospital-acquired infection. To control hospital-acquired infections, a sound management system should be established. In addition, medical staff, especially newcomers and interns, must receive strengthened training to improve their knowledge of hospital-acquired infection. Hand hygiene and the rational use of antibacterial drugs should be emphasized.
\end{abstract}

The incidence of hospital-acquired infections have increased annually with the extensive use of hormone, immunosuppressing, and broad-spectrum antibacterial drugs. Hospital-acquired infections have become a global problem that is difficult to solve, and may influence the prognosis and increase the pain burden of patients, as well as even cause death. Hospital-acquired infections add to the workload of medical staff, cause huge economic losses to the patients, and affect the economic and social benefits provided by hospitals. This paper presents a review of the research status of the epidemiology and prevention of infections in Chinese hospitals.

\section{Pathogenic microorganisms}

The five main pathogenic microorganisms involved in hospital-acquired infections are Staphylococcus aureus, Pseudomonas aeruginosa, Acinetobacter baumannii, Klebsiella pneumonia, and Candida albicans ${ }^{[1]}$. Lower respiratory tract infections are mainly caused by Gram-negative bacilli, followed by P. aeruginosa, Escherichia coli, K. pneumonia, A. baumannii, and Enterobacter cloacae. Gram-positive cocci are relatively small and include $S$. aureus, $S$. epidermidis, and enterococcus. C. albicans and mycoderma are the main fungal species associated with hospital-acquired infections ${ }^{[2]}$. Pathogenic bacteria associated with hospital-acquired infection should be definitively identified based on microbial culture. However, in China, etiology inspections are rarely conducted and most patients with infection are given drugs based on the clinician's experience instead of etiology and drug sensitivity. In addition, specimen collection is not standardized. Antibiotics with poor effects are frequently replaced, thus increasing the drug resistance of bacteria and thereby influencing the treatment effect. Therefore, clinicians should be trained in hospital-acquired infection knowledge and etiology inspections should be standardized and frequently performed. The results of the drug sensitivity test can provide scientific evidence for the clinical treatment of infectious diseases and should be used as the basis for the selection of antibiotic drugs ${ }^{[3]}$.

\section{Epidemiological characteristics of hospital-acquired infection}




\section{Susceptible locations}

In patients, hospital-acquired infections are mainly localized in the lower respiratory tract, followed by in the upper respiratory tract and surgical incision ${ }^{[4-5]}$. Respiratory infections are caused by various invasive operations and limited ward space. In addition, airborne pathogen transmission is difficult to control and easily causes cross infections; thus, respiratory tract infections rank first as hospital-acquired infections. To decrease infection rates, the natural ventilation and air quality of the inpatient area should be improved. The management of air quality in the ward, the disinfection of nursing and diagnostic supplies, and the hand hygiene of the medical staff should be improved. Patients with infection should be treated in time, and the necessary quarantine and protective measures for susceptible individuals should be adopted to decrease the incidence of respiratory infections ${ }^{[6]}$.

\section{Susceptible departments}

Many studies have shown obvious differences in the rates of hospital-acquired infection among different wards in Chinese hospitals, with the comprehensive intensive care unit (ICU) having the highest infection rate ${ }^{[7-8]}$. Some studies have identified the surgical ICU, hematology, emergency, respiratory medicine, and neurology departments as the departments that are highly susceptible to hospital-acquired infection ${ }^{[9-10]}$. Knowing the departments at high risk for hospital-acquired infection will help establish regulations for infection control and monitoring ${ }^{[7]}$. In addition, infection rates per department can decrease via the systematic training of medical staff, especially those working in high-risk department, in the knowledge of hospital-acquired infection, as well as the strict implementation of a management system and standard prevention measures for infection.

\section{Susceptible groups}

Ma Fei et al. ${ }^{[11]}$ pointed out that the incidence of infection is distributed in a V-shaped manner among patients. Infants (< 2 years old) and senior patients ( $>60$ years old) have a wide variety of infectious diseases and high rates of infection. These two groups are more susceptible to hospital-acquired infections because infants have immature immune systems and senior patients usually present with a severe condition, several basic diseases, and comparatively low immune function, as well as undergo more invasive surgeries.

\section{Route of transmission and influencing factors Hand hygiene of medical staff}

The hand hygiene of the medical staff is the most important, simplest, most effective and most economical approach to the prevention and control of hospital-acquired infections [12]. Many studies and surveys have shown that the hands of medical staff are contaminated by and transmit numerous pathogenic microorganisms. The infections that result from the direct or indirect hand transmission of pathogenic bacteria account for more than $30 \%$ of the cases of hospitalacquired infection ${ }^{[13]}$ Therefore, hand hygiene has received increased attention from the World Health Organization and the Worldwide Patient Security Association. These organizations have identified hand hygiene as an important performance indicator and strongly encourage the development of hand hygiene in various medical institutions at all levels.

\section{Use of antibiotics}

The use of antibiotics is an important measure in clinical treatment. However, the unreasonable application of antibiotics will result in recurrent infection and cause the emergence of resistant strains and adverse reactions. Antibiotic treatment should be pertinent and rational. To ensure the rehabilitation of the patients, a clear etiological diagnosis should be established as early as possible and should be a prerequisite for antibiotic treatment. The abuse of antibiotic drugs, especially broad-spectrum antibacterial drugs in many patients, strengthens the drug resistance of pathogenic bacteria, making individual or group bacterial infections difficult to treat and more likely to spread. Drug resistance is also a crucial factor that induces secondary and fungal infections. The use of antibiotic drugs should be regulated; methods for the identification of bacterial culture and drug sensitivity should be improved; full doses of antibiotics should be provided for a limited duration; and the preventive use of antibiotic drug should be limited. ${ }^{[14]}$ The special conditions of community hospitals and the age, weakness, and low immune function of patients with chronic diseases facilitate the transmission of hospitalacquired infections. Antibiotic drugs should be administered to patients in accordance with the indications and counterindications of the drugs. The clinical laboratory and pharmacy department should regularly publish the testing results for major pathogenic bacteria and drug sensitivity to provide clinicians with information about medication. 
Nurses should accurately execute medical advice in accordance with drug compatibility and preparation ${ }^{[15]}$. To summarize, implementing a management system and strengthening quality control will prevent the abuse of antibiotics.

\section{Control of hospital-acquired infections}

\section{Establish a management system for hospital-acquired infection}

The problem of hospital-acquired infections has become more severe with the development of modern medicine. Therefore, the management of hospital-acquired infection is essential to improve the medical quality, security, and social benefit provided by hospitals ${ }^{[16]}$. A sound organization and management network with a practical and feasible system plan should be established. The knowledge of the medical staff about hospital-acquired infections should be strengthened. The participation and quality of all the medical staff should be improved. Medical staff should supervise, monitor, analyze, and react to hospital-acquired infection and its relevant factors, as well as closely concentrate on drug resistance and the distribution of infections among departments. Pertinent control and monitoring measures should be implemented and standardized to effectively control hospital-acquired infections ${ }^{[17]}$.

Supervision alone is insufficient in controlling hospitalacquired infections ${ }^{[18]}$. Some studies have identified that the improper behaviors of the medical staff cause most hospitalacquired infections ${ }^{[19]}$. Some members of the hospital staff, particularly technicians in assisting departments, have a poor understanding of occupational protection and hand hygiene. During practical work, which involves exposure to the bodily fluids and blood of the patients, some staff members do not wear masks and caps. In addition, some members of the medical staff do not wash their hands in accordance with sterile operation. Medical specimens are discarded without following standard procedure, and medical wastes are not clearly categorized. Therefore, the supervision of community hospitals should be strengthened ${ }^{[20-21]}$. Although the majority of the medical staff in community hospitals is aware of hospital-acquired infection, which they positively prevent and control in their practical work, some problems remain in the implementation of control measures ${ }^{[22]}$. Members of the medical staff generally have insufficient knowledge about occupational protection and hand hygiene. Although most community hospitals have established a management institution for hospital-acquired infection, management remains insufficient ${ }^{[15]}$. To further decrease the incidence and control infection in community hospitals, the medical staff should be informed about and trained in hospital-acquired infection. In addition, the management of hospital-acquired infection in community hospitals should be improved. In the meantime, etiology inspections should be regulated and enforced because the results of the drug sensitivity test provide the scientific basis for the diagnosis and treatment of infections ${ }^{[3]}$.

\section{Improve the understanding of the medical staff}

Any hospital-acquired infection is directly or indirectly related with the medical staff. Regardless of the physician, nurse, technician or logistics, medical personnel and interns all influence the incidence of hospital-acquired infection. In recent years, numerous materials and training methods have been provided for the control and management of hospital-acquired infections. However, some members of the medical staff have incomplete knowledge of the regulations and systems of hospital-acquired infection given the rapid development of novel sanitizers, sterile methods, and monitoring techniques. Studies have shown that $80 \%$ of the medical staff do not wash their hands or wear masks before and after sterile operation or treating critical patients; $60 \%$ of the medical staff do not follow the six-step hand-washing method, do not wear masks before entering the ward, and do not wash hands after contacting the patients' case history and personal items. In practical medical work, the physician, nurse, or medical technician might follow nonstandard operation. Therefore, the medical staff should improve their awareness of self-protection and proactively control hospitalacquired infections ${ }^{[23]}$.

Zhou Ying et al. ${ }^{[23]}$ reported that the cognition of hospitalacquired infection improves with increasing work experience. Medical staff members with extensive work experience have a better mastery of preventing hospital-acquired infections, indicating that the knowledge of hospital-acquired infection is mainly derived from continuing education. Therefore, all nurses, particularly newly hired nurses, should receive regular educational training on hospital-acquired infection. Training on hospital-acquired infections should be a crucial course for student nurses. Many studies have shown that interns have insufficient knowledge of the concept and 
techniques of isolation, sterilization, and disinfection, as well as of the risk factors, self-protection, and research development in hospital-acquired infection. Before their clinical internship, medical students should be trained in the knowledge of occupational infection. Doing so will provide medical students with a general understanding of common occupational hazards in the hospital and prevention countermeasures ${ }^{[24]}$. In different phases of medical internship, training on effective measures against occupational hazards should be provided to strengthen the awareness of medical students. The incidence of hospitalacquired infection could be decreased by enhancing the supervision, control, active participation, and prevention awareness of the medical staff ${ }^{[25]}$.

\section{Emphasize hand hygiene}

The hands of the medical staff, especially those of nurses, are important routes of hospital-acquired infection because nurses are in frequent contact with patients. Nurses perform each treatment using their hands, which are contaminated with pathogens that cause hospital-acquired infections ${ }^{[26}$ -27]. Yang Chunhong et al. ${ }^{[24]}$ reported that nurses and doctors usually have higher compliance with hand hygiene than interns, who usually have high error rates in washing methods. Therefore, educational lectures on hospitalacquired infection and occupational prevention that emphasize hand hygiene should be regularly presented.

Hand hygiene and disinfection are important measures for preventing and controlling hospital-acquired infections. Given that hands are easily contaminated and overlooked, hand hygiene is the basis for controlling hospital-acquired infection. Zhang Xiuping etc. ${ }^{[28]}$ reported that the incidence of hospital-acquired infections can be decreased through knowledge training, regular hand hygiene activity, the strict regulation of hand skin hygiene and disinfection in accordance with Hospital-acquired Infection Management Standard, and the promotion of rapid hand sanitization and reasonable glove wear.

Microbial inspection in rate remains limited in China. In most cases, patients are treated with drugs based on clinical experience instead of examination and drug sensitivity test. In addition, specimen collection is not standardized. Most hospital wards have poor air quality and weak ventilation, thus facilitating the rapid growth and transmission of pathogenic bacteria. Only $80 \%$ of the medical staff practices aseptic operation by wearing a mask and washing their hands before and after treating critical patients. Some members of the medical staff do not follow the six-step hand-washing method, do not wear masks before entering wards, and do not wash their hands when contacting the case history and patients' items. In addition, many patients blindly use antibiotics, especially broad-spectrum antibiotics, thus increasing the drug resistance of bacteria and causing refractory and epidemic infections. In actual medical work, doctors, nurses, and medical inspectors all follow different nonstandard operation. Therefore, clinicians should be trained in the relevant knowledge of hospital-acquired infection and etiology inspection should be standardized. The medical staff should improve their self-protective awareness and hand hygiene activity should be performed regularly. In the meantime, the use of anti-bacterial drugs should be regulated. The correct dose of antibacterial drugs should be provided for a short duration. The blind, frequent replacement of antibiotics and the prophylactic use of antibiotics should be limited. Training on the relevant knowledge should be strengthened. Monitoring, disinfection, and isolation could help control hospital-acquired infections.

\section{Declarations}

\section{Acknowledgements}

No.

\section{Competing interests}

The author declares that he has no competing interest.

\section{Authors' contributions}

WL He made the literature analysis and wrote, discussed and revised the manuscript of this review.

\section{References}

1 Chen $\mathrm{X}, \mathrm{Hu}$ Y. Pathogenic bacteria distribution characteristics and tendency of hospital-acquired infection in comprehensive hospitals, Chinese Journal of Nosocomiology. 2011, 21(3):575-577.

2 Gould IM. Antibiotic policies to control hospital-acquired infection. J Antimicrob Chemother, 2008, 61(4):763-765.

3 Liang G, Li S, Qin Y, et al. Application research on the pathogenic bacteria distribution characteristics and drug resistance dynamic changing in antibiotic drug of the hospital-acquired infection. Clinical use of drug, 2013, 6(1):79-80.

4 Zhao H. Inspection analysis on the prevalence rate of hospital-acquired 
infection, Public Medical Forum Magazine, 2012, 16(10): 1332-1334.

5 Sun S, Ni G. Inspection analysis on the prevalence rate of hospitalacquired infection. Chinese Journal of Noscomiology, 2012, 22(10): 2026-2028.

6 Ding M. Analysis of risk factors and control of hospital-acquired infection. Modern Hospitals, 2009, 9(9):93-94.

7 Zhang W, Liu G, Li X, et al. Inspection analysis on the prevalence rate of hospital-acquired infection during 2009-2011. Chinese Journal of Noscomiology, 2012, 22(17): 3075-3077.

8 Li W, Deng M. Inspection analysis on the prevalence rate of hospitalacquired infection in 2010. West China Medical Journal, 2012, 27(11): 1673-1675.

9 Zhao J, Liu Y, Xing Y, et al. Descriptive research on the hospital-acquired infection in comprehensive hospitals. Chinese Journal of Noscomiology, 2013, 23(8):1780-1782.

10 Singh AK, Sen MR, Anupurba S, et al. Antibiotic sensitivity pattern of the bacteria isolated from nosocomial infections in ICU.J Commun Dis, 2002, 34(4): 257-263.

11 Ma F, Li D. Research status of the hospital-acquired infection epidemiology. Liberation Army Prevention Medicine Magazine, 2006, 24(5): 386-389.

12 Bilski B, Kosinski B. An analysis of selected hands hygiene habits in selected population of nursing staff. Medycyna pracy, 2007, 58(4): 291.

13 Janet R, Refik S, Kurt W, et al. Patterns of hand-washing behavior and visits to patients on a general medical ward of health care workers. Infect Control and Hosp-Epidemiol, 2004, 25:198.

14 Wang A. Reasonable usage of antibiotic drug to control the hospitalacquired infection. Clinical Rational Drug Use, 2011, 4(2B):103.

15 Zhang Q, Zheng M, Zhu L. Cognition and management inspection analysis on community medical staff hospital-acquired infection. Chinese Journal of Noscomiology, 2012, 22(11): 2381-2383.

16 Yang K, Ren Y, Zhou H, et al. Hospital-acquired infection management progress of emergency intensive care unit. Chinese Journal of Noscomiology, 2009, 19(9):1169-1170.
17 Zou H. Management discussion on hospital-acquired infection. Chinese Journal of Convalescent Medicine, 2011, 20(6):571-573.

18 Yang J. Problems and countermeasures in the management of community hospital-acquired infection. Chinese and Foreign Medical Research, 2011, 9(36):174-175.

19 Torres Viera C, Dembry LM. Approaches to vancomycin-resistant Enterococci. Curr Opin Infect Dis, 2004,17(6):541-547.

20 Zhang Y, Tian X, Shi J, et al. Problem and countermeasures of the hospital-acquired infection in 96 community health service institution. China Practical Medical, 2013, 8(7): 265-266.

21 Han Y, Zhang J, Wu B. Management methods and measures of the community hospital-acquired infection. Chinese Journal of Noscomiology, 2011, 21(23):5016.

22 Korniewicz DM, El-anasri M. Exploring the factors associated with hand hygiene compliance of nurses during routine clinical practice. Appl Nurs Res, 2010, 23(2):86-90.

23 Zhou Y, Chen X, Deng L, et al. Hospital inspection analysis of infection cognition level and protective behavior of medical staff. Chinese Journal of Noscomiology, 2012, 22(20): 4556-4558.

24 Yang C, Qiu H, Li Q, et al. Cognition status and influencing factor inspection analysis on Intern hospital-acquired infection knowledge. Chinese Journal of Noscomiology, 2012, 22(17): 3790-3792.

25 Li Q, Liao T, Xiong L, et al. Knowledge cognition status research of assistant intern on hospital-acquired infection. China Medical Herald, 2012, 9(22):133-137.

26 Liang $Q$, Hand hygiene research progress of medical staff. Chinese Journal of Disinfection, 2011, 28(2): 219-221.

27 Li J, He B, Tian X. Importances of hand hygiene in the prevention of hospital-acquired infection. Chinese Journal of Modern Drug Application, 2010, 4(2):236-237.

28 Zhang X, Lu Y, Ren J. Hand hygiene inspection analysis status of the knowing-trusting-practice of the nurses in operating room. Journal of Nursing Science, 2011, 26(6):52-53. 